\title{
Indications for Liver Transplantation in the Cyclosporine Era
}

\author{
Robert D. Gordon, M.D.,* \\ Byers W. Shaw, Jr., M.D.,† \\ Shunzaburo Iwatsuki, M.D. $\ddagger$ \\ Carlos O. Esquivel, M.D., Ph.D., § \\ and Thomas E. Starzl, M.D., Ph.D.\|
}

Organ transplantation became practical when long-term allograft survival was achieved in renal transplant recipients treated with a combination of azathioprine and prednisone. ${ }^{4,28}$ The introduction of antilymphocyte globulin (ALG) as a short-term agent for the prevention or treatment of acute rejection further improved results, ${ }^{27}$ but the morbidity and mortality of cadaver renal transplantation remained significant. ${ }^{14}$

Advances in liver transplantation have closely paralleled events in the evolution of cadaveric kidney transplantation. From March 1, 1963, until February 29, 1980, 170 patients received liver transplants at the University of Colorado under conventional immunosuppression with azathioprine (or cyclophosphamide) and high-dose steroids, to which antilymphocyte globulin (ALG) was almost always added. ${ }^{26}$ The 1 - and 5-year survivals were 32.9 per cent and 20.0 per cent, (Fig. 1). As of July 1, 1985, only 29 patients ( 16.5 per cent) remained alive, including 14 adults and 15 children, 10 of whom have survived 10 or more years. Transplantation of the liver with conventional immunosuppression was never able to achieve the success required to move it from the realm of experimental surgery to accepted clinical therapy.

Cyclosporin-A (cyclosporine) was introduced to transplantation based

From the Department of Surgery, University Health Center of Pittsburgh, University of Pittsburgh, Pittsburgh, Pennsylvania

*Assistant Professor of Surgery

†Assistant Professor of Surgery

$\ddagger$ Assistant Professor of Surgery

$\S$ Assistant Professor of Surgery

$\|$ Professor of Surgery

This study was supported by research grants from the Veterans Administration and by Grant No. Am-29961 from the National Institutes of Health, Bethesda, Maryland. 


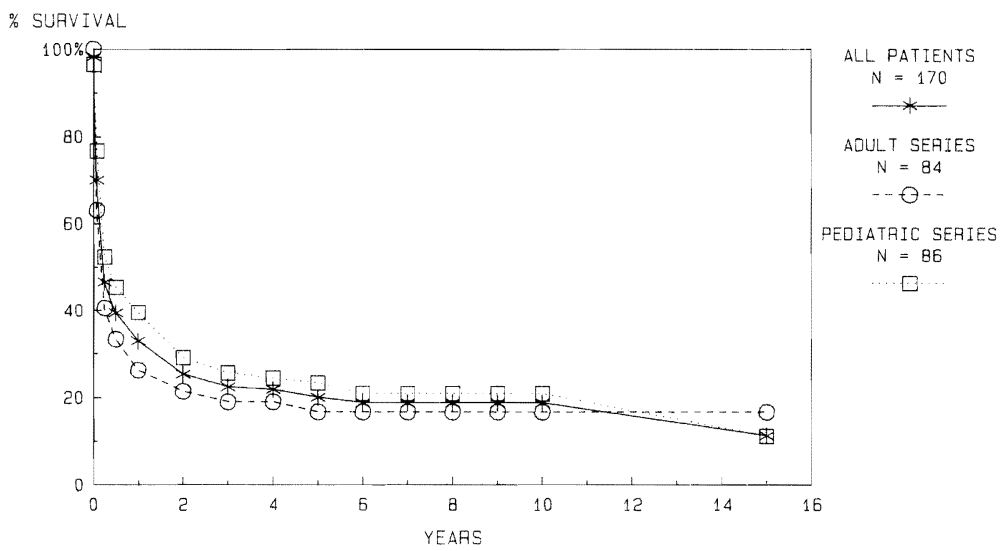

Figure 1. Survival of 170 liver transplant recipients treated with conventional immunosuppression with azathioprine (or cyclophosphamide), high-dose steroids, and ALG between March 1, 1963, and February 29, 1980, at the University of Colorado.

on the experimental work of Borel and colleagues ${ }^{1}$ and the first clinical trials done by Calne and associates. ${ }^{2} \mathrm{~A}$ high patient mortality, a disquieting incidence of spontaneous lymphomas, and cyclosporine-induced nephrotoxicity raised questions about the safety of the drug. Combination with low doses of prednisone permitted use of reduced doses of cyclosporine, with reduction in nephrotoxicity and patient mortality. ${ }^{18,23,30}$ The lymphomas are now known to be associated with Epstein-Barr virus infection ${ }^{3,23,29,30}$ and to resolve when immunosuppression is withdrawn or reduced. ${ }^{29}$

Cyclosporine-steroid therapy was introduced to the liver transplant program at the University of Colorado on March 1, 1980, and continued when the program transferred to the University of Pittsburgh. From March 1, 1980, through December 31, 1984, 313 patients, including 177 adults and 136 children, have received primary orthotopic liver transplants in the Colorado-Pittsburgh series under the cyclosporine-prednisone regimen. A clinical trial with OKT3 monoclonal antibody (Ortho Pharmaceuticals, Raritan, New Jersey) for the treatment of acute rejection began in October 1984. One- and five-year actuarial survivals are 69.7 per cent and 62.8 per cent, respectively (Fig. 2). This dramatic improvement over results obtained with conventional immunosuppression has led to acceptance of liver transplantation as a therapeutic operation ${ }^{12}$ and has catalyzed a proliferation of liver transplant programs in the United States and abroad. ${ }^{25}$

There have been changes in the indications for liver transplantation since the introduction of cyclosporine (Figs. 3 and 4) ${ }^{20}$ Biliary atresia has been the most common indication for liver replacement since 1980. In adults, primary biliary cirrhosis and sclerosing cholangitis are more common indications for liver transplantation, and alcoholic cirrhosis and primary liver tumors have declined. Acceptance of transplantation as the treatment of choice for nearly all causes of liver failure has broadened patient selection and increased patient referral to the transplant center. 


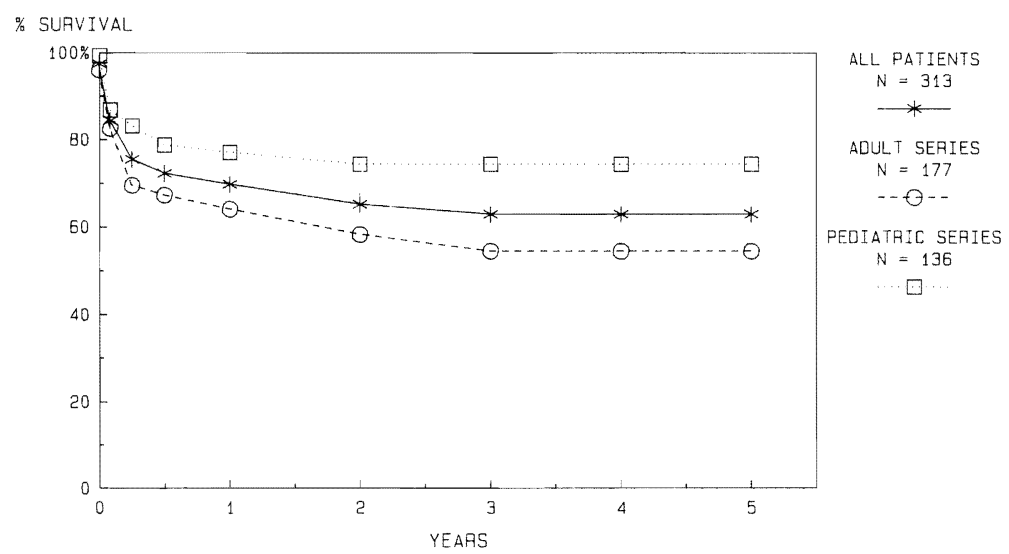

Figure 2. Survival (life table method) is shown for 313 liver transplant recipients treated with cyclosporine and low-dose steroids between March 1, 1980, and December 31, 1984, at the University of Colorado and the University of Pittsburgh. Treatment of acute rejection epidoses with OKT3 monoclonal antibody (Ortho Pharmaceuticals, Raritan, New Jersey) was begun in 1984 .

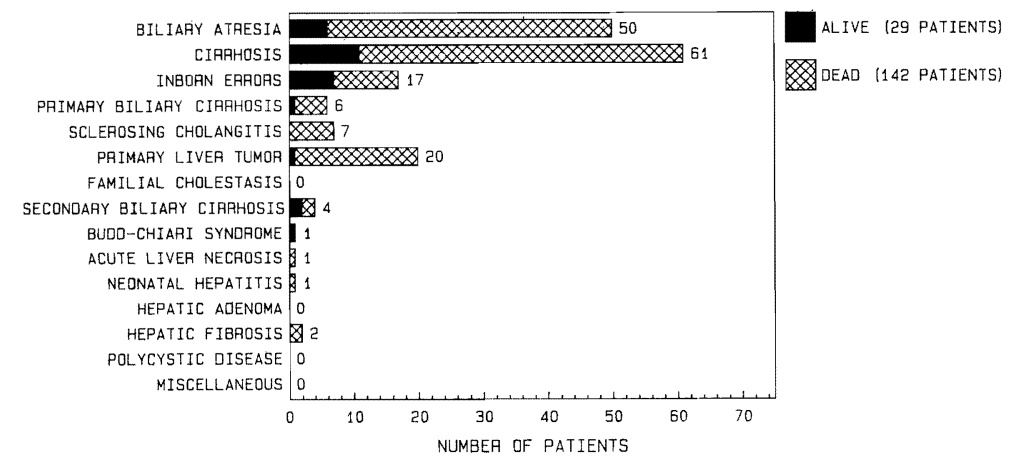

Figure 3. Indications are shown for 170 liver transplants done with conventional immunosuppression (azathioprine and high-dose steroids) from March 1, 1963, to February 29, 1980. Twenty-nine patients are still living. 


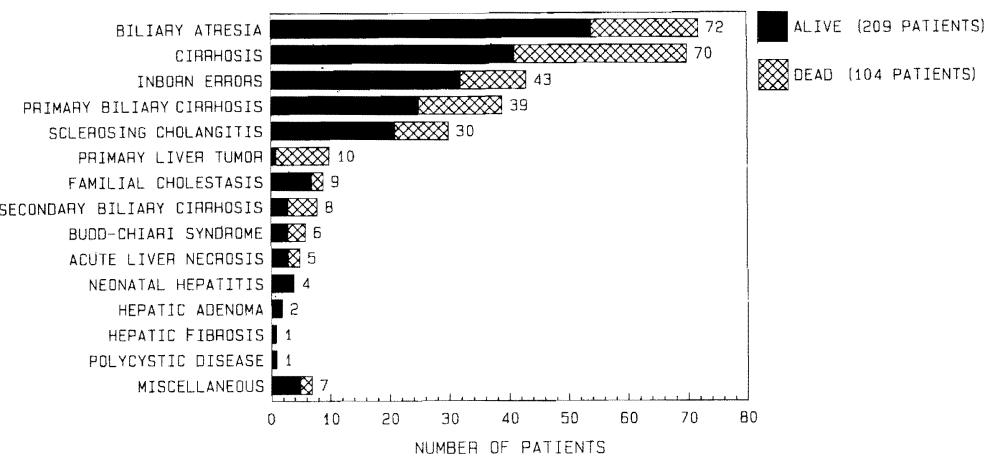

Figure 4. Indications for liver transplantation are shown for 313 patients treated with cyclosporine and low-dose steroids betwen March 1, 1980, and December 31, 1984. Two hundred nine patients are still living, including 106 of the 177 (59.9 per cent) adults and 103 of the 136 (75.7 per cent) children in the series.

\section{BILIARY ATRESIA}

Biliary atresia has been the reason for transplantation in $72(52.9$ per cent) of the 136 children receiving transplants. Fifty-four of these children are alive at least 6 months after transplantation. One- and five-year actuarial patient survival rates are 76.2 per cent and 73.7 per cent, respectively (Fig. 5). Patients operated on at age 2 years or less have not done as well as older patients. One- and five-year survival rates in these patients are 64.8 per cent and 57.6 per cent, respectively.

Biliary atresia is cured by liver transplantation, and the excellent

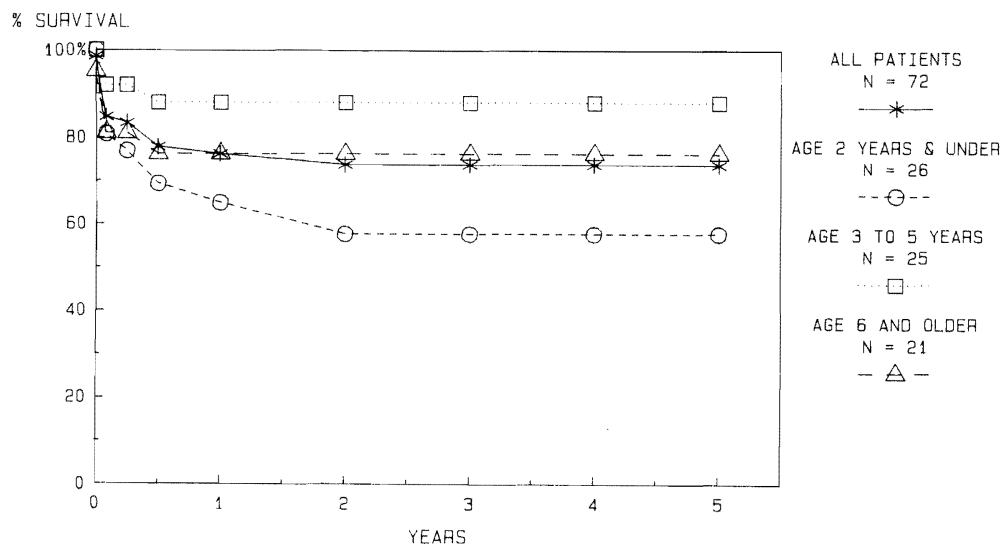

Figure 5. Actuarial (life table) survival after liver transplantation with cyclosporine and prednisone is shown for 72 patients with biliary atresia. Children under 2 years of age often receive transplants in worse condition and with a higher incidence of technical complications than older children. 
patient survival now obtainable makes liver replacement the treatment of choice for this disorder. The greatest problem limiting treatment of biliary atresia is the limited availability of very small pediatric donors. Most of the patients undergoing liver transplantation for biliary atresia have had at least one portoenterostomy (Kasai) procedure. A successful biliary diversion can stabilize the patient and buy valuable time. However, previous abdominal surgery, especially in the right upper quadrant, makes hepatectomy in a transplant recipient more difficult. A single biliary diversion procedure usually will not increase the risk of transplantation, but multiple attempts at revision of biliary drainage and the creation of stomas can make transplantation an arduous and dangerous undertaking.

\section{INBORN ERRORS OF METABOLISM}

Forty-three patients, including 29 children and 14 adults, have received liver transplants for correction of inborn errors of metabolism. Actuarial survivals at 1 and 5 years are both 74.4 per cent (Fig. 6). Twenty-three patients are living more than 1 year after surgery, and there have been no deaths after the first year. Most of these transplants were performed for cirrhosis related to the metabolic disorder. In general, these patients are excellent candidates for transplantation, as few have had previous surgery, and if the disease is detected early enough, the patients can receive the transplants before they develop severe liver failure.

\section{Alpha-1-Antitrypsin Deficiency}

Twenty-seven patients have received transplants for alpha-1-antitrypsin deficiency. This disorder is associated with development of a progressive macronodular cirrhosis and obstructive pulmonary disease. Transplantation is curative and results in conversion of the PiZZ to PiMM phenotype and

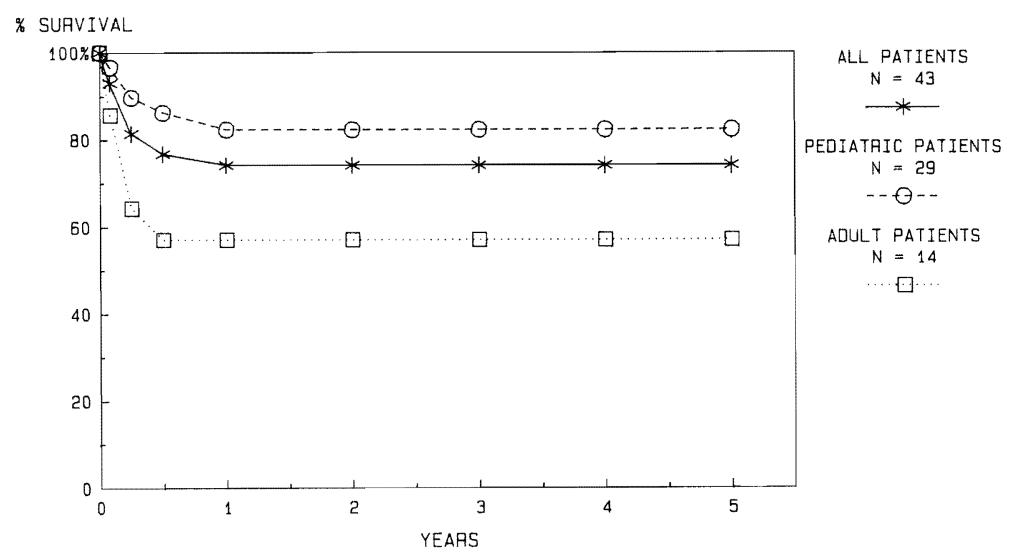

Figure 6. Actuarial (life table) survival is shown for 43 patients receiving liver transplants with cyclosporine and prednisone for inborn errors of metabolism. Except for patients in advanced hepatic failure with coma, survival for patients with this group of diseases has been excellent. 
normalization of serum alpha-1-antitrypsin levels. Actuarial survival is 70.4 per cent at both 1 and 5 years. Fourteen patients are living 1 year or more after operation, and there have been no deaths beyond 6 months after transplantation.

\section{Wilson's Disease}

Seven patients have received transplants for Wilson's disease, a disorder associated with reduced copper excretion, decreased serum ceruloplasm, and excessive copper deposition in both liver and brain. Five patients are surviving. Liver transplantation is curative and is indicated when hepatic involvement becomes signficant. The first presentation of this illness can be acute hepatic failure and hemolytic crisis. It is best to allow recovery from the acute hemolytic crisis before proceeding with transplantation, but recently a 13-year-old girl in irreversible acute hepatorenal failure underwent a successful transplant operation. Patients presenting in deep coma have the poorest prognosis.

\section{Tyrosinemia}

Hereditary tyrosinemia, which results in a progressive cirrhosis, necessitated transplantation in five patients, four of whom remain alive 13 to 44 months after surgery. The abnormal metabolic by-products of tyrosine are carcinogenic, and patients with tyrosinemia are at high risk of developing hepatocellular cancer. Transplantation should be performed before malignancy develops. At the time of transplantation a 5-year-old boy with tyrosinemia was found to have a tumor extending into the portal vein. Although all gross tumor was removed at surgery and the patient received a course of chemotherapy postoperatively, he died from recurrent cancer within 8 months of transplantation. Apparently, the hepatocellular cancer can persist independent of the metabolic disorder. Nevertheless, all of the survivors had hepatomas that have not recurred.

\section{Other Metabolic Errors}

Two patients have received transplants for glycogen storage disease and two patients for hemochromatosis. All four patients are living 8 to 41 months following liver replacement.

Recently a 12-year-old girl with homozygous familial hypercholesterolemia complicated by intractable angina and cardiac failure received a simultaneous heart and liver transplant. The heart transplant was performed to replace the severely damaged heart that had already undergone several surgical procedures. The liver, which was anatomically normal, was replaced to correct the underlying metabolic disorder. Now, more than 15 months after surgery, the patient has serum cholesterol levels that have remained less than $300 \mathrm{mg}$ per $\mathrm{dl}$ compared with pretransplant levels of more than $1000 \mathrm{mg}$ per $\mathrm{dl}$. Liver transplantation is likely to become the treatment of choice for many metabolic deficiencies that have their origin in the liver.

\section{CIRRHOSIS}

Seventy patients, including 13 children and 57 adults, have received liver transplants for postnecrotic cirrhosis. Most of these patients have the 


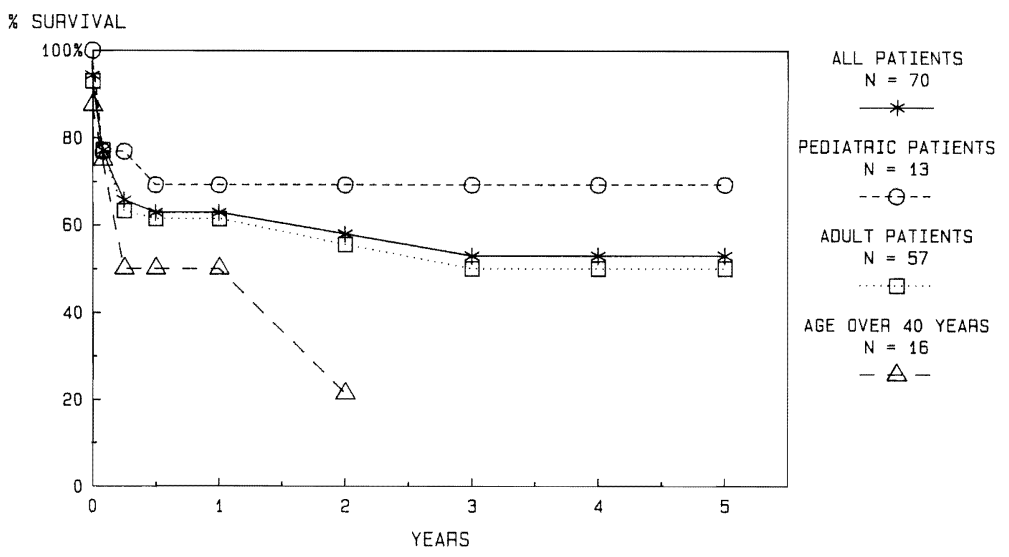

Figure 7. Actuarial (life table) survival is shown for 70 patients receiving liver transplants with cyclosporine and prednisone for postnecrotic cirrhosis. Patients over 40 years of age have not done as well as younger patients.

non A, non B variety of chronic hepatitis. One and five-year actuarial survivals are 62.8 per cent and 52.9 per cent. Children fare better than adults, and patients over 40 years of age have the worst prognosis (Fig. 7). Only 6 of the 16 patients over 40 survived the first year after transplantation.

Five patients with positive serology for hepatitis $\mathrm{B}$ surface and $\mathrm{E}$ antigen were treated with human antihepatitis B immune globulin and with Hepatavax-B vaccine. All five patients either retained or reverted to their original hepatitis serology. Two of these patients have died with recurrent hepatitis. Although this is a limited experience, it suggests that patients with positive hepatitis B serology remain at significant risk of recurrence, especially if they have $\mathrm{E}$ antigenemia.

Two recent cases have further stimulated interest in transplantation for hepatitis. A 24-year-old surgical resident who contracted acute hepatitis from patient contact presented in severe hepatic failure and stage IV coma. An emergency liver transplant was performed, and the patient made a rapid recovery. Since surgery he has remained antibody positive but surface antigen negative, and he is likely to be at low risk of recurrence. A 15year-old hemophiliac with chronic active hepatitis acquired as a complication of factor VIII transfusions required liver transplantation for progressive cirrhosis. ${ }^{10}$ After surgery he converted to an antigen-negative serology, and his new liver is making normal amounts of factor VIII. The liver transplant, which was performed for cirrhosis, may have cured his hemophilia. Longer follow-up is needed to assess the risk of recurrent hepatitis.

Because there have been no specific markers for non A, non B hepatitis, it is difficult to assess the risk of recurrence in this group of patients. Two patients developed what may have been an acute episode of hepatitis, but both recovered. In general, results in this group of patients have been encouraging, and transplantation should be performed before disabling complications set in. 


\section{PRIMARY BILIARY CIRRHOSIS}

Primary biliary cirrhosis (PBC) is a chronic, nonsuppurative, destructive cholangitis of unknown etiology. ${ }^{7}{ }^{17}$ It is an uncommon but not rare disorder that most often afflicts middle-aged women. The disease is associated with a variety of immunologic abnormalities and is considered to be an autoimmune disorder. ${ }^{6,32}$ A variety of other diseases have been reported in association with $\mathrm{PBC}$, including scleroderma, rheumatoid arthritis, thyroiditis, interstitial pneumonitis, lupus erythematosus, renal tubular acidosis, Sjögren's syndrome, and non-Hodgkin's lymphomas.

PBC usually presents as jaundice, fatigue, pruritis, and hepatomegaly in middle-aged women. Laboratory studies show cholestasis and positive antimitochondrial antibodies. Liver biopsy shows degeneration or necrosis of interlobular bile ducts, granulomas, and mononuclear cell infiltrates. Metabolic bone disease is a common and serious complication of the disease.

A variety of medical treatments have been tried to control this disease. The most extensive experience has been with D-pencillamine, ${ }^{11}$ but recently chlorambucil, colchicine, and cyclosporine have been introduced in clinical trials. Although clinical trials are continuing, results of drug therapy have been disappointing and have had little effect on survival.

Thirty-nine patients have received liver transplants for PBC. Most have been women in the fifth or sixth decade of life who had severe jaundice, ascites, esophageal varices, and advanced bone disease. An increase in the progression of jaundice, recurrent bleeding from esophageal varices, recurrent encephalopathy, and severe hepatic osteomalacia are the usual reasons for referral to the transplant center. Operation is often not technically difficult in these patients, since most have had minimal previous surgery and the large liver can be easy to remove. However, the advanced age and debilitated state of many of these patients complicate postoperative recovery. Disabling bone disease may result in a long, difficult recuperation.

Actuarial survivals at 1 and 5 years are both 64.1 per cent (Fig. 8). Twenty-four patients are now living 1 to 6 years after transplantation, and there have been no patient deaths beyond 6 months after transplantation. Most patients' antimitochondrial antibody titers remain positive after transplantation. It is difficult to distinguish histologically chronic allograft rejection from PBC. However, so far no case of recurrent $\mathrm{PBC}$ has been conclusively documented in any of the patients with transplants, in contrast to the experience of Neuberger and colleagues ${ }^{13}$ of Cambridge.

In the absence of effective medical therapy, liver transplantation is the treatment of choice for patients entering the advanced stages of primary biliary cirrhosis. Referral to the transplant center before the patient becomes severely debilitated and avoidance of unnecessary abdominal surgery, especially in the right upper quadrant, can optimize the chances of successful transplantation. Operation for diagnosis is inappropriate.

\section{SCLEROSING CHOLANGITIS}

Sclerosing cholangitis is a chronic, cholestatic disease of unknown etiology. 3, 9, 31 It is characterized by inflammation and fibrosis of the biliary 


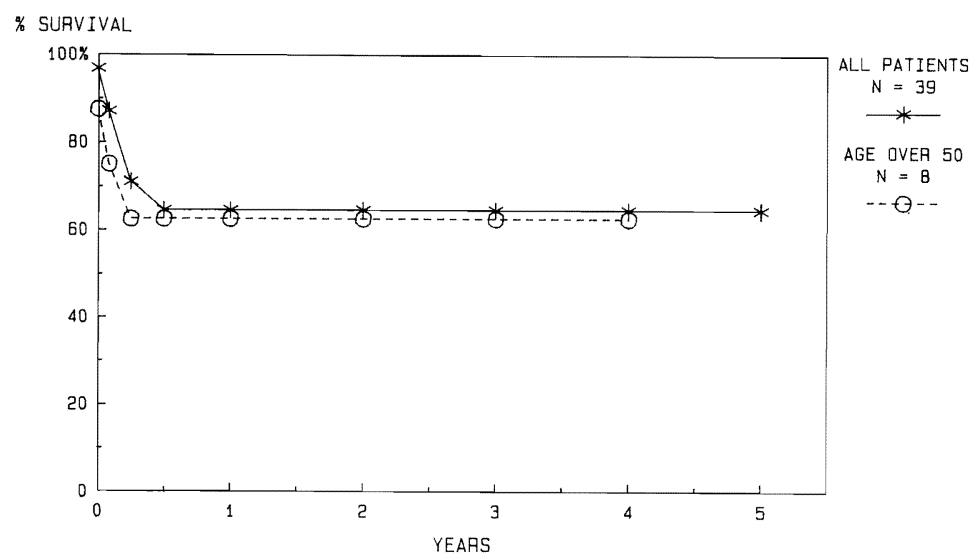

Figure 8. Actuarial (life table) survival is shown for 39 patients receiving liver transplants with cyclosporine and prednisone for primary biliary cirrhosis. Advanced age has not influenced survival in this group of patients but may prolong recuperation.

system. Although the disease may localize to selected portions of the intrahepatic or extrahepatic biliary tree, sclerosing cholangitis is often a diffuse process. Liver biopsy demonstrates bile duct proliferation, periductal fibrosis and inflammation, ductal obliteration, and loss of bile ducts.

Sclerosing cholangitis may occur as a primary disease or in association with other disorders, especially inflammatory bowel disease. There is strong evidence that sclerosing cholangitis is a premalignant condition that predisposes to the development of bile duct cancer.

Sclerosing cholangitis is an incurable and eventually fatal disease. Medical treatment is aimed mainly at the control of complications. Surgery for sclerosing cholangitis is indicated only in special circumstances. Operation for diagnosis is unnecessary, except when differentiation of sclerosing cholangitis from adenocarcinoma of the bile duct cannot be accomplished by other means. Operative biliary diversion and drainage procedures should be limited to selected patients with well-localized extrahepatic disease. Percutaneous biliary decompression is now the procedure of choice for most patients with localized obstructions. Biliary tract surgery has little to offer the patient with diffuse disease and can complicate liver replacement. The early experience with liver transplantation in these patients suffered because many patients had been subjected to multiple operations to treat extrahepatic biliary obstructions and because referral for transplantation was often delayed until patients were moribund from hepatic failure.

Survival after liver transplantation for sclerosing cholangitis has improved. Thirty patients have now received transplants for this disease with 1- and 5- year actuarial survivals of 69.1 per cent (Fig. 9). Twenty-one patients are alive 6 months to 5 years after surgery, and there have been no deaths beyond 1 year after transplantation.

Selection of patients with sclerosing cholangitis requires careful individualization. Duct cell tumors must be considered, especially in patients presenting with primary sclerosing cholangitis or a long history of the 


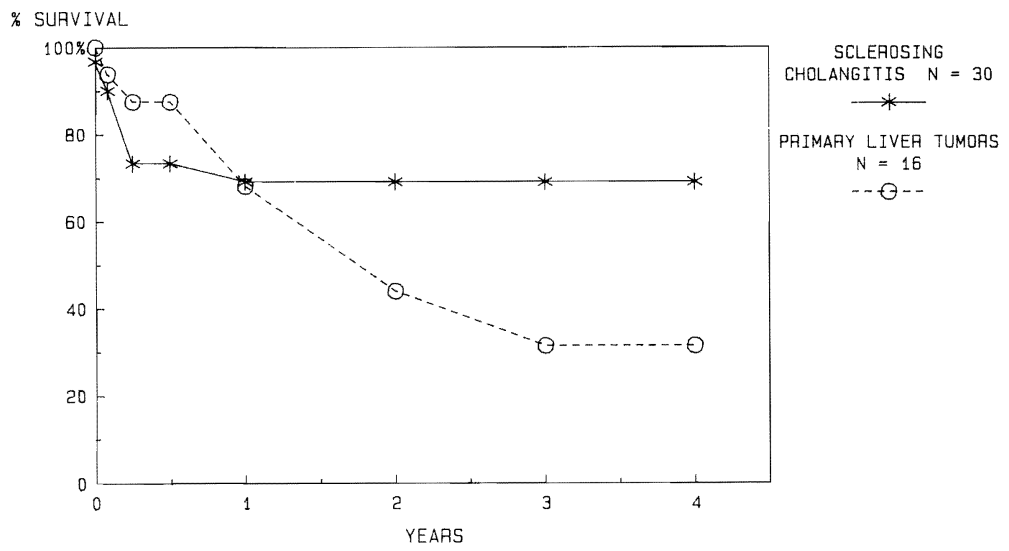

Figure 9. Actuarial (life table) survival is shown for 30 patients receiving liver transplants for sclerosing cholangitis and 16 patients receiving transplants for primary liver malignancies.

disease. Patients with colitis are at increased risk of developing colonic malignancy when under immunosuppression after liver transplantation. Whenever indicated, proctocolectomy should be deferred for 3 to 6 months after liver replacement. The risk of recurrence of sclerosing cholangitis and the risk of bile duct cancer after transplantation are not yet known.

\section{PRIMARY LIVER MALIGNANCY}

The earliest liver transplants using conventional immunosuppression were performed for liver tumors that could not be removed by conventional hepatic resection. These patients are usually excellent operative candidates, and early survival after transplantation has been good. However, recurrence of tumor within 1 year of liver transplantation has been high, resulting in a high patient mortality. ${ }^{5}$

Thirty-six patients with primary hepatic malignancy have been treated by transplantation. Twenty of these patients were treated before the introduction of cyclosporine. Only 6 patients survived beyond 6 months, and all but one died within 54 months. All 19 patients lost had metastatic disease at the time of death. The lone survivor was originally thought to have a sarcoma of undertermined histologic type with miliary metastatic lesions at the time of transplantation. She is alive 8 years later, with no evidence of active tumor growth. Re-examination of her tumor years later has led to a revised diagnosis of low-grade malignancy, if it is malignant at all. ${ }^{15}$

Sixteen patients with transplants received cyclosporine (Fig. 9). Only seven patients are living. Two surviving patients have known recurrences, and a third patient, now seven months from operation, had residual tumor in regional lymph nodes.

There are two exceptions to the generally grim prognosis in this group of cancer patients treated with transplantation. First, 12 of 13 patients 
received transplants for primary liver tumors incidental to other causes of end-stage liver disease have survived from 4 months to more than 15 years since transplantation. Second, six of the seven patients with the fibrolamellar variant of hepatocellular cancer ${ }^{16}$ have survived for more than 1 year. Metastases have developed in 4 of the 6 patients, two of whom have died. The two patients living with known recurrent fibrolamellar tumors have been followed for 19 and 38 months. Liver transplantation offers more effective palliation and possible cure in the patient with this variant of liver cancer.

Generally, our experience with primary hepatic cancer has been disappointing and suggests that liver transplantation must be combined with other therapeutic modalities to offer effective treatment to most patients with this illness. ${ }^{5}$

\section{MISCELLANEOUS DISEASES}

Seven patients with Budd-Chiari syndrome have received transplants. One patient on azathioprine and three patients on cyclosporine have survived. Two other patients survived 16 and 20 months after transplantation. One died following retransplantation for chronic rejection, and the other died of recurrence after anticoagulation was interrupted in preparation for a liver biopsy. Two of the three surviving patients required extensive thromboembolectomy of the vena cava and iliofemoral systems or of the portal vein at the time of transplantation, and all three patients have been maintained on permanent anticoagulation with Coumadin.

Of nine patients who received transplants for familial cholestasis, seven have survived 6 months to 4 years after operation. The two deaths occurred within 3 months of transplantation. Four patients received transplants for neonatal hepatitis. All are surviving 6 months to 4 years after surgery.

Only three patients with alcoholic cirrhosis have undergone transplant operations since the introduction of cyclosporine. Two died on the operating table. We have been reluctant to perform transplantation in this group of patients because of the long waiting list of candidates with non-self-inflicted diseases who are much better risks for surgery and because of the difficulty in determining which patients have truly reformed their lifestyles. However, increasing numbers of patients with alcoholic cirrhosis will be treated with liver replacement.

\section{RETRANSPLANTATION}

Twenty-one liver retransplantations were done using conventional immunosuppression with azathioprine. ${ }^{5}$ Only four patients lived more than 6 months, and all were dead within 14 months of retransplantation.

About 20 per cent of the patients who undergo liver transplantation will require another transplant. ${ }^{19}$ Sixty-eight patients have undergone a second transplantation from March 1, 1980, through December 31, 1984. Thirty-one patients are living (Fig. 10). As with primary transplants, survival has been better in children than in adults (Fig. 11). 


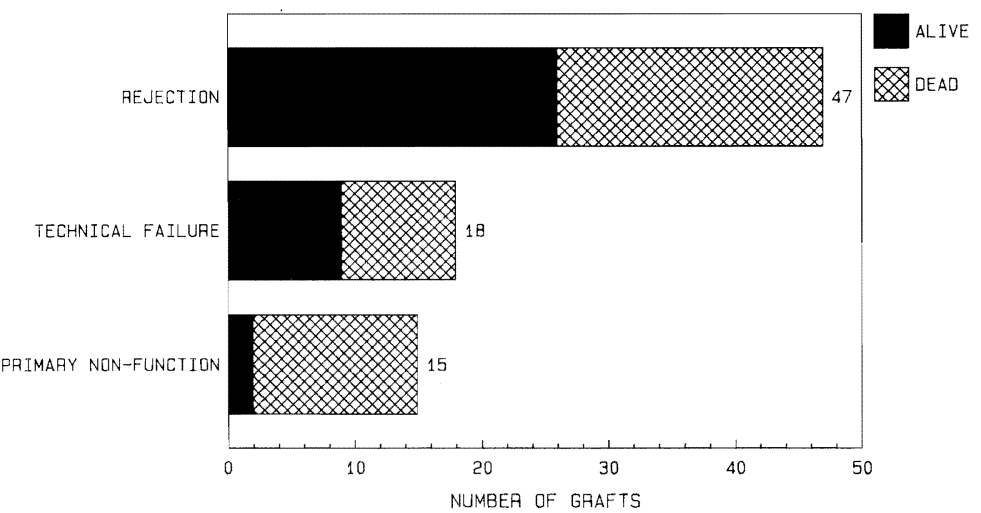

Figure 10. Indications for liver retransplantation. Eighty liver retransplantations were performed using cyclosporine and prednisone between March 1, 1980, and December 31, 1984. Sixty-eight patients received a second liver transplant, and 12 patients received a third liver transplant. Rejection was the most common reason for retransplantation ( 58.8 per cent).

The indication for retransplantation has a definite bearing on survival (Fig. 12). Patients requiring retransplantation for primary graft failure decompensate rapidly, and retransplantation must be done urgently. Only 2 of the 13 patients requiring a second graft for primary nonfunction of a first transplant have survived. On the other hand, 21 of 39 patients requiring a second graft for rejection and 8 of 16 patients requiring a second graft for technical complications have survived.

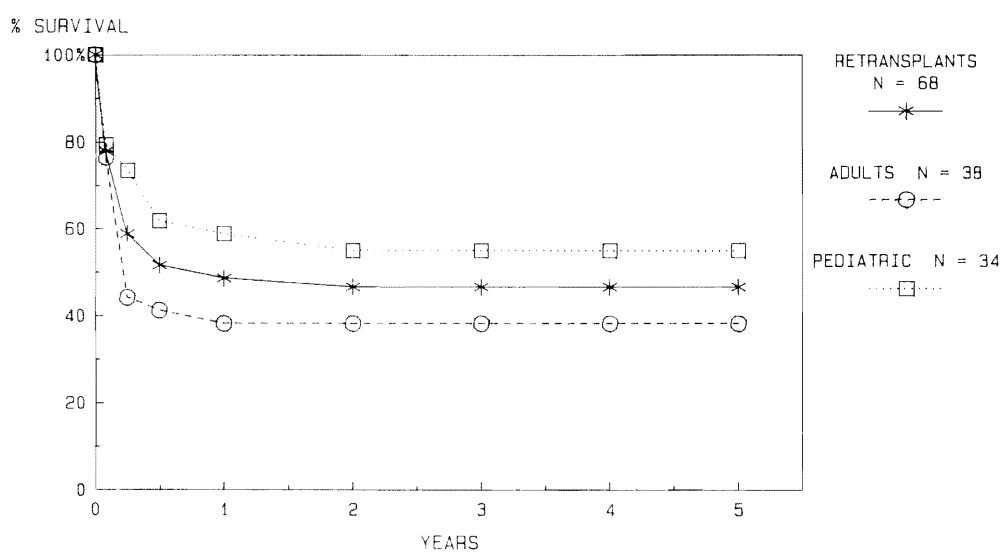

Figure 11. Actuarial survival (life table method) after retransplantation with cyclosporine and prednisone from March 1, 1980, to December 31, 1984. Survival is better in children than in adults. Fifteen ( 39.5 per cent) of the 38 adults receiving retransplantations are surviving compared with 22 (52.4 per cent) of the 42 children. 


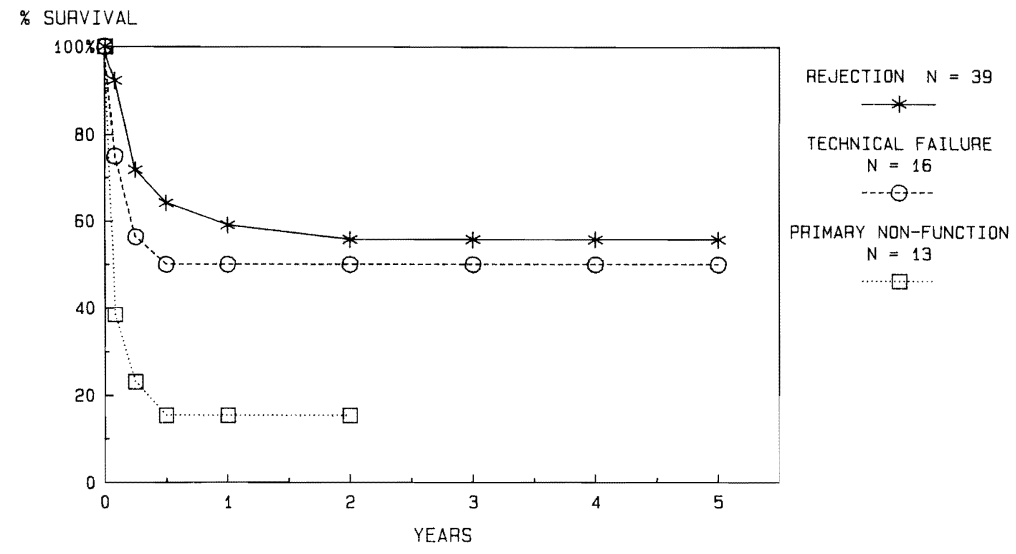

Figure 12. Actuarial survival after liver retransplantation based on indication for graft replacement from March 1, 1980, to December 31, 1984. Over one half of the patients receiving retransplantations for rejection and nearly one half of those with retransplantations because of technical failure are surviving. Retransplantation for primary graft failure has had a high mortality rate.

Technical complications requiring a second graft are more common in children (38.2 per cent) than in adults (8.8 per cent). Hepatic artery thrombosis accounts for nearly all technical problems requiring retransplantation. It usually presents in one of three clinical patterns: fulminant liver failure (acute hepatic gangrene), delayed biliary leak (bile duct necrosis), or relapsing bacteremia. ${ }^{33}$ Patients presenting with hepatic gangrene or bile duct necrosis require retransplantation, as do most patients with relapsing bacteremia who develop liver abscess. However, a small number of children appear to tolerate hepatic artery thrombosis without development of liver abscess and can be sustained with antibiotics until enough arterial collaterals develop. This small group of patients does not require retransplantation.

About 10 per cent of patients will have an accelerated form of rejection. These patients may require urgent retransplantation. Most of the time, however, rejection is a gradual process, and retransplantation can be done under semielective conditions. Survival after retransplantation for rejection of a first graft has been good. Twenty-one of the 39 ( 53.8 per cent) requiring retransplantation for rejection of the first graft have survived.

Twelve patients have required a third transplant. Six of these patients have survived. Five of the six patients who rejected both a first and a second transplant have survived after a third transplant.

Successful retransplantation with cyclosporine in nearly 50 per cent of the patients needing such treatment has contributed significantly to the improved results now being achieved. However, retransplantation adds greatly to the cost of care. The average hospital cost from 1983 to 1984 for pediatric liver transplant patients who required only one graft was about $\$ 85,000$. The average hospital cost for pediatric patients requiring retransplantation was nearly $\$ 200,000 .^{20}$ 


\section{CONCLUSION}

Liver transplantation is now the treatment of choice for a variety of diseases leading to irreversible liver failure in children and adults. Immunosuppressive therapy based on cyclosporine-prednisone has tipped the scales more than any other factor, but improvements in organ procurement techniques and in the recipient operation have also contributed.

The extent to which patient selection can influence results of transplantation is hard to assess. As results have improved, there is increased justification for avoidance of invasive and less effective alternative treatments and for earlier referral of patients to the transplant center. It is also clear that liver transplantation is of limited, if any, benefit for most patients with primary hepatic malignancy and for moribund patients in the terminal phase of advanced liver failure.

Liver transplantation is costly, and the supply of donor organs is less than the demand for them. Should prioritization of candidates for liver transplantation be based on the probability of success or on the urgency of need? Except at extreme ends of the spectrum, it is difficult now to quantify risk. Shaw and colleagues ${ }^{22}$ recently assessed risk factors in a series of 118 adults who received primary orthotopic liver transplants using a scoring system designed to distinguish high-risk patients from low-risk patients. A sigmoid curve was found to best describe the relationship between risk score and patient survival. Since many of the patients were found to have scores falling between the inflection points of the curve, it was difficult for most patients to predict the likelihood of success based on perioperative assessment of risk. Many patients who might seem to be at high risk have an excellent chance for survival in the hands of a skilled transplant team.

Given the uncertainties of predicting outcome based on risk, we continue to select patients for liver transplantation based on the urgency of need but with congnizance of those extreme circumstances in which transplantation would be wasteful and inappropriate. This places us under constant pressure not to neglect the progression of illness in patients who have accrued months of waiting on the candidate list and enables us to offer transplantation on a basis that physicians, patients, families, and the general public can accept as equitable.

\section{SUMMARY}

One hundred seventy orthotopic liver transplants were performed under conventional immunosuppression with azathioprine and steroids with 1- and 5-year survivals of 32.9 per cent and 20.0 per cent, respectively. Since the introduction of cyclosporine-prednisone therapy in March 1980, 313 primary orthotopic liver transplants have been performed. Actuarial survivals at 1 and 5 years have improved to 69.7 per cent and 62.8 per cent, respectively.

Biliary atresia is now the most common indication for liver replacement. In adults, primary biliary cirrhosis and sclerosing cholangitis have become more common indications for transplantation, and alcoholic cirrhosis and primary liver malignancy as indications have declined. Early enthusiasm 
for liver transplantation in patients with hepatic cancer has been tempered by the finding that recurrence is both common and rapid. An increasing number of patients with inborn errors of metabolism originating in the liver are receiving transplants, including patients with Wilson's disease, tyrosinemia, alpha-1-antitrypsin deficiency, glycogen storage disease, familial hypercholesterolemia, and hemochromatosis. Survival in this group of patients has been excellent ( 74.4 per cent at 1 and 5 years). A hemophiliac who received a transplant for postnecrotic cirrhosis has survived and may have been cured of his hemophilia.

About 20 per cent of patients require retransplantation for rejection, technical failure, or primary graft failure. Only four of the patients receiving retransplants under conventional immunosuppression survived beyond 6 months, and all died within 14 months of retransplantation. Sixty-eight patients have received retransplants under cyclosporine-prednisone. Thirtyone patients are surviving, all for at least 1 year. Six of the twelve patients requiring a third transplant are alive 2 to 3 years after the primary operation. An aggressive approach to retransplantation in the patient with a failed graft is justified.

\section{REFERENCES}

1. Borel, J. F., Feurer, C., Gubler, H. U., et al.: Biological effects of Cyclosporin A: A new antilymphocytic agent. Agents Actions, 6:468, 1976.

2. Calne, R. Y., Rolles, K., White, D. J. G., et al.: Cyclosporin A initially as the only immunosuppressant in 34 recipients of cadaveric organs, 32 kidneys, 2 pancreases, and 2 livers. Lancet, 2:1033, 1979.

3. Hanto, D. W., Gajl-Peczalska, K. J., and Frizzera, G.: Epstein-Barr virus (EBV) induced polyclonal and monoclonal B-cell lymphoproliferative disease occurring after renal transplantation. Ann. Surg., 198:356, 1983.

4. Hume, D. M., Magee, J. H., Kauffman, H. M., Jr., et al.: Renal homotransplantation in man in modified recipients. Ann. Surg., 158:608, 1963.

5. Iwatsuki, S., Gordon, R. D., Shaw, B. W., Jr., et al.: Role of liver transplantation in cancer therapy. Ann. Surg., 202:401-407, 1985.

6. James, S. P., Vierling, J. M., and Strober, W.: The role of the immune response in the pathogenesis of primary biliary cirrhosis. Semin. Liver Dis., 1:322, 1981.

7. Kapelman, B., and Schaffner, S.: The natural history of primary biliary cirrhosis. Semin. Liver Dis., 1:273, 1981.

8. LaRusso, N. F., Wiesner, R. H., Ludwig, J., et al.: Primary sclerosing cholangitis. N. Engl. J. Med., 310:899, 1983.

9. Lefkowitch, J. H.: Primary sclerosing cholangitis. Arch. Intern. Med., 142:1157, 1982.

10. Lewis, J. H., Bontempo, F. A., Spero, J. A., et al.: Liver transplantation in a hemophiliac. (Letter to the Editor.) N. Engl. J. Med., 312:1189, 1985.

11. Matloff, D. S., Alpert, E., Resnick, R. N., et al.: A prospective trial of D-pencillamine and primary biliary cirrhosis. N. Engl. J. Med., 306:319, 1982.

12. National Institutes of Health Consensus Development Conference Statement: Liver Transplantation-June 20-23, 1983. Hepatology, 4(Suppl. 1):107S, 1984.

13. Neuberger, J., Portmann, B., Macdougall, B. R., et al.: Recurrence of primary biliary cirrhosis after liver transplantation. N. Engl. J. Med., 306:1, 1982.

14. Opelz, G., Mickey, M. R., and Terasaki, P.: HLA matching and cadaver kidney transplant survival in North America: Influence of center variation and presensitization. Transplantation, 23:490, 1977.

15. Weiss, S. W., and Enzinger, F. M.: Epithelioid hemangioendothelioma: A distinctive vascular tumor often mistaken for a carcinoma. Cancer, 50:970, 1982. 
16. Peters, R. L.: Pathology of hepatocellular carcinoma. In Okuda, K., and Peters, R. L. (eds.): Hepatocellular Carcinoma. New York, John Wiley, 1975.

17. Roll, J., Boyer, J. L., Barry, D., et al.: The prognostic importance of clinical and histologic features in asymptomatic and symptomatic primary biliary cirrhosis. N. Engl. J. Med., $308: 1,1983$.

18. Rosenthal, T. J., Hakala, T. R., Iwatsuki, S., et al.: Cadaveric renal transplantation under cyclosporine-steroid therapy. Surg. Gynecol. Obstet., 157:309, 1983.

19. Shaw, B. W., Jr., Gordon, R. D., Iwatsuki, S., et al.: Hepatic retransplantation. Transplant. Proc., 17:264, 1985.

20. Shaw, B. W., Jr., Gordon, R. D., Iwatsuki, S., et al.: Transplantation of the liver. In Moody, F. C. (ed.): Surgical Treatment of Digestive Disease. Chicago, Year Book Medical Publishers, 1986. pp. 425-445.

21. Shaw, B. W., Jr., Martin, D. J., Marquez, J. M., et al.: Venous bypass in clinical liver transplantation. Ann. Surg., 200:524, 1984.

22. Shaw, B. W., Jr., Wood, R. P., Gordon, R. D., et al.: Influence of selected patient variables and operative blood loss on six month survival following liver transplantation. Semin. Liver Dis., 5:385-393, 1985.

23. Starzl, T. E., Hakala, T. R., Rosenthal, T. J., et al.: Variable convalescence and therapy after cadaveric renal transplantation under Cyclosporin A and steroids. Surg. Gynecol. Obstet., 154:819, 1982.

24. Starzl, T. E., Hakala, T. R., Shaw, B., Jr., et al.: A flexible procedure for multiple cadaveric organ procurement. Surg. Gynecol. Obstet., 158:223, 1984.

25. Starzl, T. E., Iwatsuki, I., Shaw, B. W., Jr., et al.: Orthotopic liver transplantation in 1984. Transplant. Proc., 17:250, 1985.

26. Starzl, T. E., Iwatsuki, S., Van Thiel, D. H., et al.: Evolution of liver transplantation. Hepatology, 2:614, 1982

27. Starzl, T. E., Marchioro, T. L., Porter, K. A., et al.: The use of heterologous antilymphoid agents in canine renal and liver homotransplantation and human renal homotransplantation. Surg. Gynecol. Obstet., 124:301, 1967.

28. Starzl, T. E., Marchioro, T. L., and Waddell, W. R.: The reversal of rejection in human renal homografts with subsequent development of homograft tolerance. Surg. Obstet. Gynecol., 117:385, 1963.

29. Starzl, T. E., Nalesnik, M. A., Porter, K. a., et al.: Reversibility of lymphomas and lymphoproliferative lesions developing under cyclosporine-steroid therapy. Lancet, $1: 583,1984$

30. Starzl, T. E., Weil, R., III, Iwatsuki, S., et al.: The use of cyclosporin A and prednisone in cadaver kidney trnasplantation. Surg. Gynecol. Obstet., 151:17, 1980.

31. Summerfield, J. A.: Primary sclerosing cholangitis. Postgrad. Med. J., 59(Suppl. 4):99, 1983.

32. Thomas, H. C.: Potential pathogenetic mechanisms in primary biliary cirrhosis. Semin. Liver Dis., 1:338, 1981.

33. Tzakis, A. G., Gordon, R. D., Shaw, B. W., Jr., et al.: Clinical presentation of hepatic artery thrombosis after liver transplantation in the cyclosporine era. Transplantation, 40:667-671, 1986 .

Thomas E. Starzl, M.D.

Department of Surgery

University Health Center of Pittsburgh

3601 Fifth Avenue

Room 103 Falk Clinic

Pittsburgh, Pennsylvania 15213 